\title{
A Smart Stick for Assisting Blind People
}

\author{
Ashraf Anwar ${ }^{1}$, Sultan Aljahdali ${ }^{2}$ \\ ${ }^{1,2}$ Faculty of Computers and Information Technology, Taif University, P.O. Box 888, 21974 Taif, SaudiArabia
}

\begin{abstract}
In this paper, we introduce a smart stick system for assisting blind people. The smart stick comes as a proposed solution to enable visually impaired people to find difficulties in detecting obstacles and dangers in front of them during walking and to identify the world around. The system is designed to act like an artificial vision and alarm unit The system consists of five sensors: ultrasonic sensor, IR sensor, water sensor, fire sensor, and light (LDR) sensor, microcontroller (Arduino Uno R3) to receive the sensor signals and process them to short pulses to the Arduino pins where buzzers, vibrator and voice alarms are connected. GPS navigation in the Mobile can be used to guide the blind for new places and unfamiliar places. The blind man uses an earphone to listen to the navigation directions that are coming from the GPS and buzzer alarm to warn by sound. We seek in our project to provide a smart stick affordable and suitable for most blind people, and also it is light in weight. It can be made available to all segments of the society and their families who need them.
\end{abstract}

Keywords: SMART STICK, BLIND PEOPLE, ARDUINo UNO, ULTRA SONIC SENSORS, HEAT SENSOR, IR SENSOR, BUZZER, VIBRATOR

\section{Introduction}

Visually impaired persons have difficulty to interact and feel their environment. They have little contact with surrounding. Physical movement is a challenge for visually impaired persons, because it can become tricky to distinguish where he is, and how to get where he wants to go from one place to another. To navigate unknown places he will bring a sighted family member or his friend for support. Over half of the legally blind people in the world are unemployed. Because limited on the types of jobs they can do. They have a less percentage of employment. They are relying on their families for mobility and financial support.

Their mobility opposes them from interacting with people and social activities. In the past different systems are designed with limitations without a solid understanding of the non-visual perception. Some of the systems are only for indoor navigations, and has no hurdle detection and determining location feature in outdoor environment. Researchers have spent the decades to develop an intelligent and smart stick to assist and alert visually impaired persons from obstacles and give information about their location [1-6].

Over the last decades, research has been conducted for new devices to design a good and reliable system for visually impaired persons to detect obstacles and warn them at danger places. There are some systems which has some deficiencies.

A Navbelt was developed by Shovalet. al [7], an obstacle avoidance wearable portable computer which is only for indoor navigation. Navbelt was equipped with two modes, in the first one the system information was translated to audio in different sounds. One sound for free for travel direction and other for blocked, it was difficult for the person to differentiate the sounds. Other problem was the system would not know the user momentary position.

A stick for distance measurement using infrared sensors, have introduced by S. Innet and N. Ritnoom [8] which is a complex and time wasting process. The stick has different vibration modes for different range which is difficult for a blind to differentiate, it needs time for training. The stick informs the person clearly at dangerous stage which conveys less information and safety. The stick has no location and positioning features. J.Na [9] proposed an interactive guide system for indoor positioning, which can't detect the obstacles and hurdles. The system is not suitable for the outdoor activities.

Our project aims to design and implement of an intelligent and cheap stick with Global Positioning System (GPS) for the visually impaired peoples, which will detect the obstacle and hurdle in the path and also determine the position and location through GPS coordinates

\section{1- System Architecture:}

\section{Methodology}

The proposed system design of the smart stick, as shown in Fig.1 is composed of the following units: 


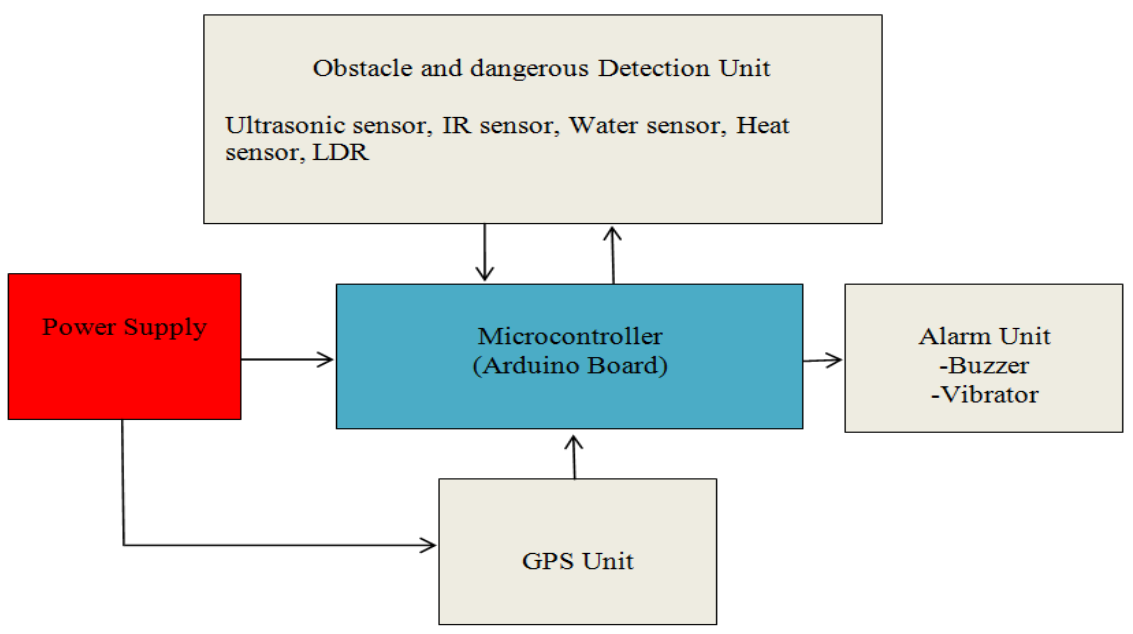

Figure1: Proposed system design of the smart stick.

\section{A. Microcontroller:}

Arduino Uno R3 Microcontroller

Arduino can control the environment by receiving input signals (Digital/Analog) and can effects its surroundings by controlling lights, relays and other devices.

The microcontroller on the board is programmed usingArduinosoftware.

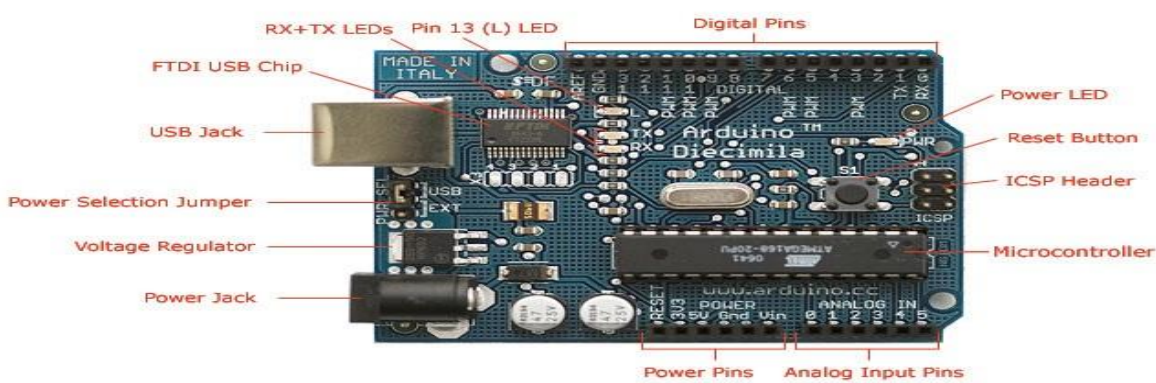

Figure 2: Arduino Uno R3 microcontroller

\section{B. Obstacles and Dangers Detection unit:}

It consists of Five sensors:

(1) Ultrasonic transducers: Generating, detecting \& processing ultrasonic signals

Ultrasonic is the production of sound waves above the frequency of human hearing and can be used in a variety of applications such as, sonic rulers, proximity detectors, movement detectors, liquid level measurement. Ultrasonic Ranging Module HC - SR04 in Figure 3

Features:

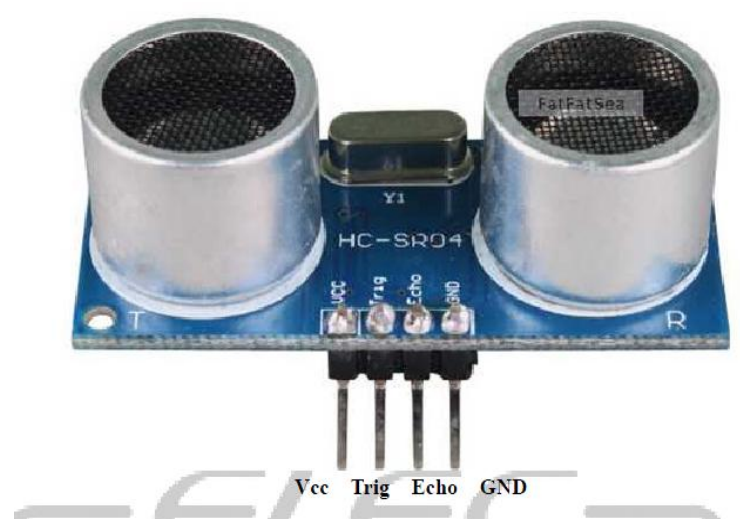

Figure3: ultra-sonic HC-SR04 
Ultrasonic ranging module $\mathrm{HC}$ - SR04 provides $2 \mathrm{~cm}-400 \mathrm{~cm}$ non-contact measurement function, the ranging accuracy can reach to $3 \mathrm{~mm}$. The modules includes ultrasonic transmitters, receiver and control circuit. The basic principle

of work:

- Using IO trigger for at least 10us high level signal,

- The Module automatically sends eight $40 \mathrm{kHz}$ and detect whether there is a pulse signal back.

- IF the signal back, through high level, time of high output IO duration is the time from sending ultrasonic to returning.

Test distance $=($ high level timexvelocity of sound $(340 \mathrm{M} / \mathrm{S}) / 2$,

The Flow chart of obstacle detector using ultrasonic sensor in Figure4 which is having two parts, first part deals with the obstacle detection while the second part deals with distance measurement, and alerting the users depending on distance of the obstacle to avoid collusion. Depending on the distance of the obstacle from the person four zones are formed: far zone, near zone, close zone and danger zone. If the detected object is at 4 meter or more then it comes under far (safe) zone. If the object is found at 2 meter or more then it comes under near zone, if the object is found at 1 meter or more then it comes under close zone, and if the object is detected at less than 1 meter then it comes under danger zone. A voice instruction along with vibrating alert and a buzzer voice will be send to user at every zone to alarm him and let people around that blind person to help him.

(2) IR sensor: To detect small obstacles: pit, staircase, or stone, as it located at the lower side of the stick. After detecting the small obstacles on ground, IR sensor will send the signal to the Arduino, as result it will send a voice instruction for small obstacle available. And at the same time it will enable the buzzer for informing the blind person about presence of obstacles on ground.

(3) Water sensor: A water sensor is located at the base of the stick to have precaution against the wet surface which it can causing slipping on the floor and thus can hurt. When the water sensor comes in contact of the wet surface, it produces an electrical signal which trigger the Arduino controller. A voice instruction for wet surface is produced and also a buzzer is enabled for alarming against a wet floor.

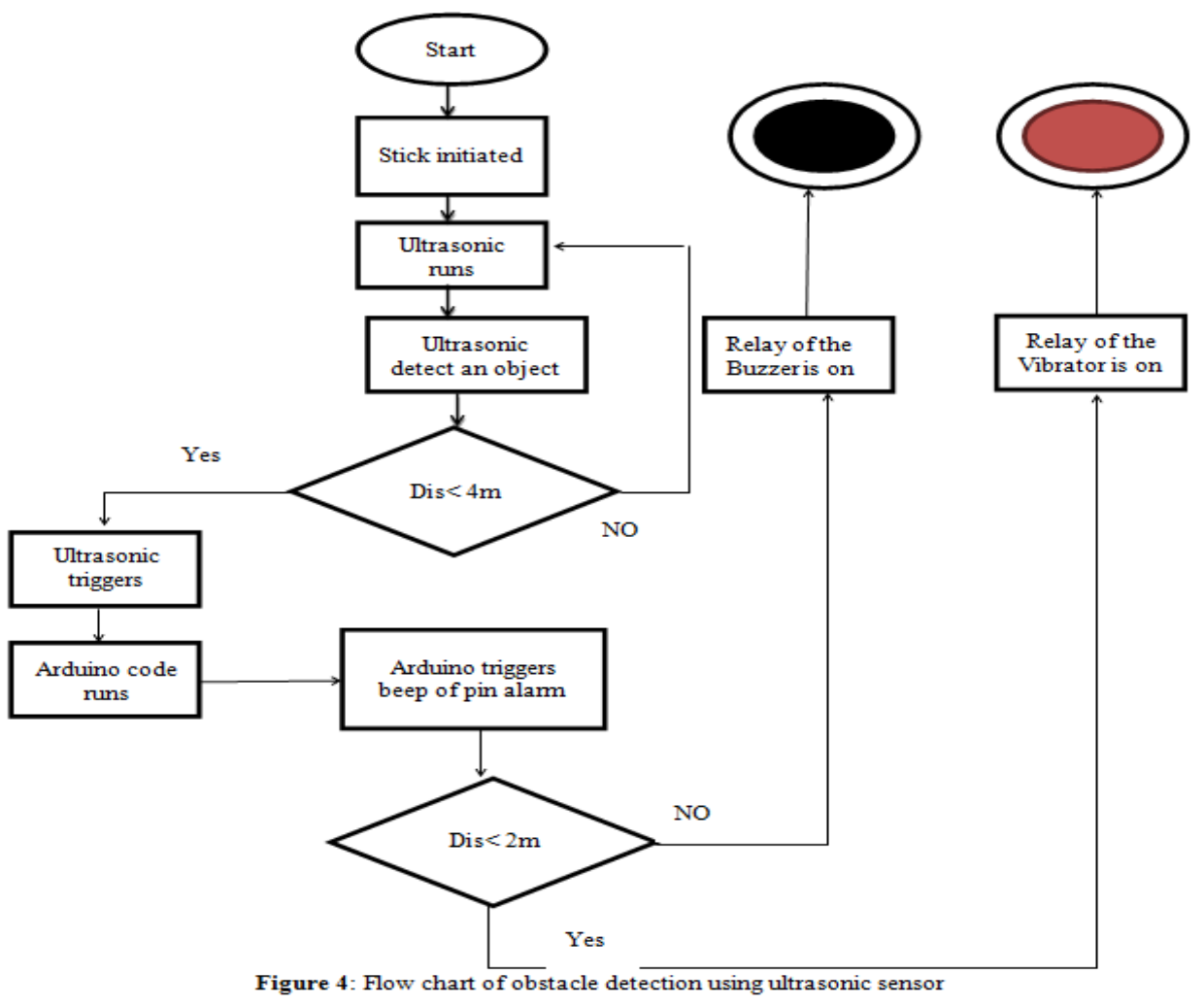

(4) Heat sensor: It is very sensitive to the heat and can detect the heat from long distance. If the sensor detects the heat radiation it will send an electrical signal to the controller and thus voice instruction will be sent to person and also the vibrator and buzzer start alarming. 
(5) LDR sensor: Light Dependent Resistor, changes its resistances due to change of the light intensity. During night, LDR will have high resistance and no current pass through it but through a LED connected parallel to it which illuminates and acts as a Flashlight, which can be easily noticed by others. It alerts people about the presence of blind person to let him to pass the way

\section{Alarm unit}

The person was informed through a vibrator and a beep sound of buzzer I t consists of two parts:

\section{(1) Buzzer}

A transducer (converts electrical energy into mechanical energy) that typically operates A buzzer is in the lower portion of the audible frequency range of $20 \mathrm{~Hz}$ to $20 \mathrm{kHz}$. This is accomplished by converting an electric, oscillating signal in the audible range, into mechanical energy, in the form of audible waves. Buzzer is used in this research to warn the blind person against obstacle by generating sound proportional to distance from obstacle

\section{(2) Vibrator}

A vibrator motor is included to enhance the overall feedback for the person who receives the warning againstobstacles closeness in different formats of vibrations.

\section{Global Positioning System (GPS) unit:}

It provides reliable positioning, navigation, and timing services to civilian users on a continuous world basis freely available to all. For anyone with GPS receiver, the system will provide location with time. The GPS based blind device with user input interfacing get alert the blind person when reaches his destination by voice.

The candidate type to use:

Skylab UART GPS Module (For Microcontroller and Arduino)

\section{Results}

1- Interfacing the Arduino:

As shown in Figure 5 connections between ultrasonic, Buzzer, and vibrator motor with the Arduino uno3 as follows:

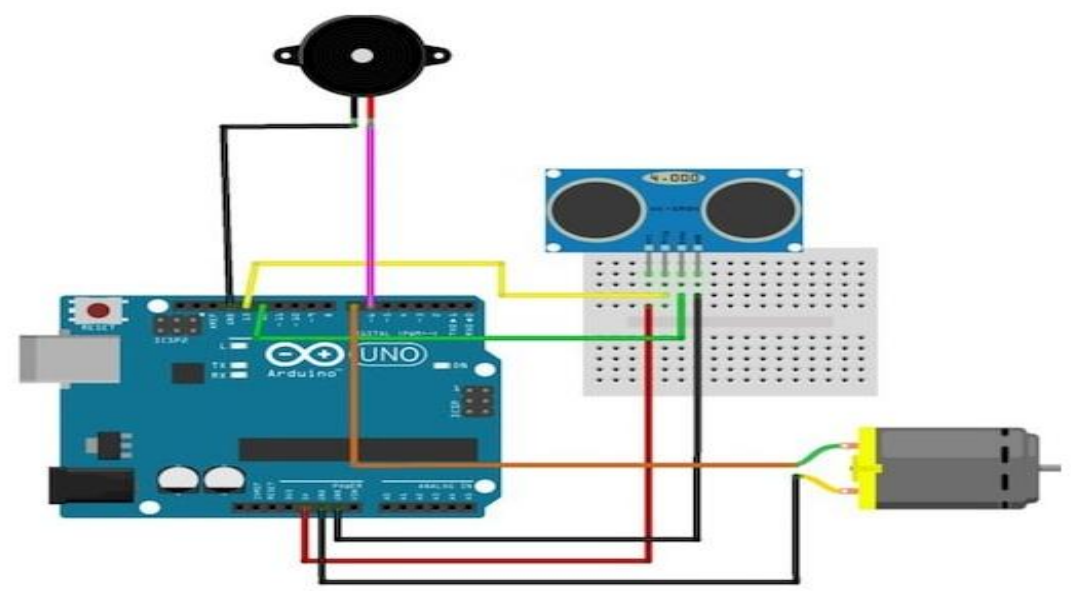

Figure 5: Arduino connections

- Ultrasonic VCC to Arduino 5v.

- Ultrasonic GND to Arduino GND.

- Ultrasonic TRIG to Arduino D12.

- Ultrasonic ECHO to Arduino D11.

- Buzzer RED to Arduino D8.

- Buzzer BLACK to Arduino GND.

- Vibrator motor pin 1 to Arduino D7.

- Vibrator motor pin 2 to Arduino GND

\section{Uploading the Sketch for the Arduino}




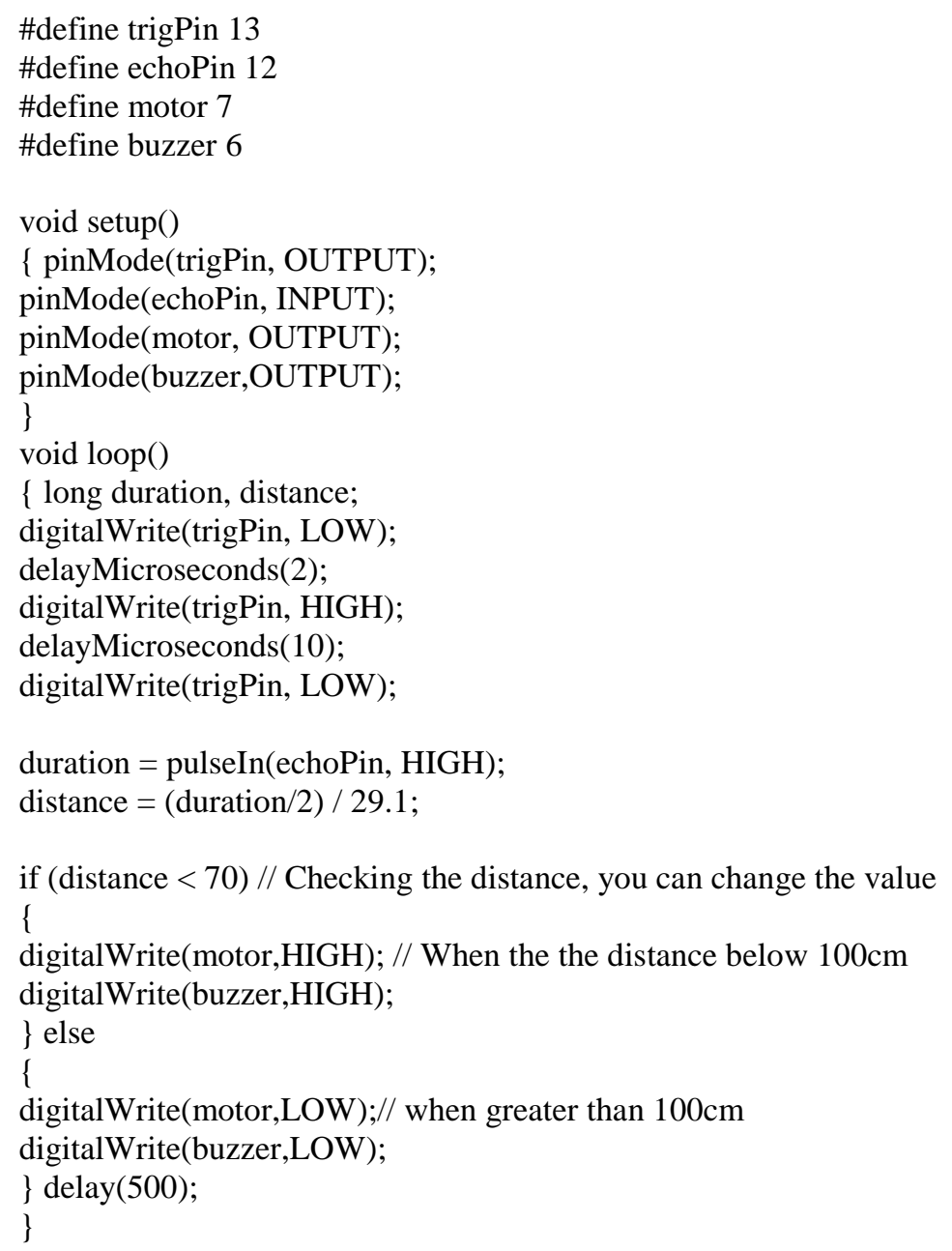

\section{Conclusion}

With the proposed architecture, if constructed with at most accuracy, the blind people will able to move from one place to another without others help, Which leads to increase autonomy for the blind. The developed smart stick that is incorporated with multiple sensors will help in navigating the way while walking and keep alarming the person if any sign of danger or inconvenience is detected.

The developed prototype gives good results in detecting obstacles paced at distance in front of the user; it will be real boon for the blind. At the same time global positioning system (GPS) can be linked with the voice stick for navigation, so that person can know his current position and distance from the destination which will be informed to users through voice instructions.

\section{References}

[1]. Sung Jae Kang, et al." Development of an Intelligent Guide-Stick for the Blind", Proceeding of the IEEE international Conference on Robotics \& Automation, 2001

[2]. Y. Kawai andF. Tomita, "A support system for visually impaired persons to understand three-dimensional visual information using acoustic interface", IEEE Conference on Pattern Recognition, Vol.3,pp.974-977,2002.

[3]. J. M. Sáez, F. Escolano, and A. Peñalver, "First steps towards stereo- based 6DOF SLAM for the visually impaired," in IEEE Conf. on Computer Vision and Pattern Recognition (CVPR), San Diego, USA,2005.

[4]. AlbertoRodriguez, et al., "Obstacle avoidance system for assisting visually impaired people", in proceeding IEEE Intelligent Vehicles Symposium Workshop, 2012.

[5]. ShrutiDambhare, et al., "Smart stick for Blind: Obstacle Detection, Artificial vision and Real-time assistance via GPS", $2^{\text {nd }}$ National Conference on Information and Communication Technology (NCICT), 2011.

[6]. Mohammad Hazzaz, et al., "Smart Walking Stick- an electronic approach to assist visually disable persons", International Journal of Scientific \& Engineering Research vol. 4, No. 10, 2013.

[7]. S.Shoval,J.Borenstein,Y.Koren,“Mobilerobotobstacleavoidanceinacomputerizedtravelaidfortheblind,"ProceedingsoftheIEEEInterna tionalConferenceonRoboticsandAutomation,May1994

[8]. S.Innet,N. Ritnoom" An Application of Infrared Sensors for Electronic White Stick" 2008 International Symposium onIntelligentSignalProcessingandCommunicationSystemsBangkok,Thailand

[9]. J.Na, "TheblindinteractiveguidesystemusingRFID based indoor positioning system, "Lecture Notesin Computer Science,SpringerPublications,vol.4061,pp.1298-1305,2006. 